in : Transportation Research Part C: Emerging Technologies, 2013 @elsevier

http://www.sciencedirect.com/science/journal/0968090X

\title{
Toward accurate localization in guided transport: combining GNSS data and imaging information.
}

\author{
Juliette Marais $^{\mathrm{a}}$, Cyril Meurie ${ }^{\mathrm{a}}$, Dhouha Attia ${ }^{\mathrm{b}}$, Yassine Ruichek ${ }^{\mathrm{b}}$, Amaury \\ Flancquart $^{\mathrm{a}}$
}

${ }^{a}$ Univ Lille Nord de France, F-59000 Lille, IFSTTAR, LEOST, F59650 Villeneuve d'Ascq

${ }^{b}$ IRTES-SET, UTBM, 90010 Belfort cedex, France

\begin{abstract}
Global Navigation Satellite Systems (GNSS) are widely spread (with Global Positioning System - GPS) in intelligent transport systems and offer a low cost, continuous and global solution for positioning. Unfortunately, urban users are often the most demanding of accurate localization but receive a degraded service because of signal propagation conditions. Several mitigation solutions can be developed. We propose, within CAPLOC project (2010-2013) to deal with inaccuracy by associating image processing techniques and signal propagation knowledge. In this paper, we focus on the contribution of image processing in more accurate position estimation. Thus, we use a laboratory vehicle, which is equipped with a fisheye camera and two GNSS receivers. The camera is located on the roof and oriented upwards to capture images of the sky. The GNSS receivers are used to obtain raw data, the position of the vehicle and the reference trajectory. The proposed approach consists in determining where satellites are located in the fisheye image, and then excluding those located in non-sky regions when calculating the position. For that, the strategy is based on an image simplification step coupled with a pixels classification. The image-based exclusion procedure is compared with the classical one based on the application of a threshold on carrier-to-noise (CN0) ratio to separate LOS and NLOS signals. Accuracy improvement is satisfying with the CNO-based method and show an improvement from $13 \mathrm{~m}$ to $4,5 \mathrm{~m}$. Image-based detection shows mixed improvements but promising: good in a static area and too harsh in another configuration of the scenario.
\end{abstract}

Keywords: Localization; Satellite; Image processing; Accuracy.

\section{Introduction}

\subsection{Context}

The policy on transportation system enhancement has recently benefited from decisions, at the European or national level with, for example, in France «Grenelle de l'Environnement ». This drives to the definition of concrete objectives in order to limit car uses and to make urban public transport more attractive and efficient. In close future, the positioning information of any actor in a transportation system will have a growing importance. This information will allow the operator to enhance its system capacity and to provide contextual information to the passengers, to the drivers or to the maintenance workers. A large panel of applications relies on positioning information: navigation, of course, but also eco-driving, toll, « pay as you drive » insurance, parking management, speed limitation, traffic signal pre-emption, etc. The position is a synchronizing element of the information system. Services like traffic management will

\footnotetext{
* Corresponding author. Tel.: +33-320-438-495; fax: +33-320-438-359

E-mail address: juliette.marais@ifsttar.fr.
} 
in : Transportation Research Part C: Emerging Technologies, 2013 @elsevier

http://www.sciencedirect.com/science/journal/0968090X

2

be enhanced and will allow more flexibility. The system will be more reliable as it benefits from a context enriched by the position. New services relying on «Context Aware » or «Ubiquitous Computing » will be deployed with more efficiency. This highlights the importance of localizing transportation systems in Intelligent Transport Systems, such as automotive vehicles and urban transport, including guided ones.

The objective of CAPLOC project is to succeed in delivering accurate positioning information, available everywhere in a global context of cost reduction. The positioning system offering the best compromise cost/simplicity/performance is today undoubtedly the satellite-based radio navigation system GPS (Global Positioning System). Moreover, in Europe, it can be enhanced by using the EGNOS (European Geostationary Navigation Overlay System) signals, to obtain a better accuracy. These solutions correspond also to the actual demands of fleet operators (including guided transport). These are expressing strong need to reduce dependence on the infrastructure, because of augmentation of vandalism acts and growing maintenance costs. Unfortunately, if the urban user of GPS is the most demanding in terms of accuracy, he has to face to a degraded service. Indeed, signal propagation conditions are strongly dependent on density of obstacles that degrades optimal reachable performances. In particular, EGNOS, being composed of geostationary satellites is received poorly in urban canyons (Kovar 2005).

In this context, the main objective of CAPLOC is to provide an innovative tool for the positioning function, relying on satellite-based technologies, GPS and EGNOS, and mitigating the difficulties linked to the constricted environment of reception. This tool has to be usable for every transport mode, and aims at becoming a key function of a large panel of mobile applications.

\subsection{Satellite-based localization in urban environments}

Global Navigation Satellite Systems (GNSS) have penetrated the transport market through applications such as monitoring of containers or fleet management. These applications do not necessarily request high availability, integrity and accuracy of the positioning system. However, new applications dealing with liability issues (toll, insurance, etc.) as well as safety-related applications (automatic guidance or control) will require more stringent performances. GPS is the only fully global operational solution for the moment and this monopole reduces the possibilities of measurement redundancy and diversity and, thus, limits the reachable performances. Since October 2009, the European Satellite-Based Augmentation System (SBAS) EGNOS enhances accuracy of the positioning. Indeed, a receiver able to use both EGNOS and GPS signals benefits from ionospheric corrections as well as parameters for protection levels computation. Unfortunately, main transport applications are used in dense urban environments, highly constraining for signal propagation. Reception conditions are bad and often impact each available satellite signal, in particular the EGNOS satellites, because of their geostationary nature. As GNSS positioning relies on propagation time measurement of at least 4 satellites simultaneously, the consequences of environmental obstructions are unavailability of the service, but also multipath reception that degrades in particular accuracy of the positioning. Indeed, Non Line Of Sight (NLOS) signals - i.e. signals received after reflections on the surrounding obstacles with no direct ray - frequently occur in densely built environments and degrade localization accuracy because of the delays observed on the propagation time measurement that create an additional error on the pseudo-range estimation.

Literature focusing on techniques for localization performance enhancement in constricted environments is abundant. The most spread rely on multi-sensor-based approaches, for which the goals are to compensate the lack of performance of GNSS by adding other sensors (odometer, Inertial Measurement Unit, etc.), that increase the system complexity. Accuracy is often enhanced by mitigating multipath error 
in : Transportation Research Part C: Emerging Technologies, 2013 @elsevier

http://www.sciencedirect.com/science/journal/0968090X

(Lentmaier 2008). Such techniques propose exclusion procedures to avoid using corrupted data (Pervan 1996). Pseudo-range reliability can be qualified by the SNR (Signal to Noise Ratio) or the elevation angle (Wang 2007). For our part, we will explore the use of image processing to determine the state of reception of every satellite.

\section{The CAPLOC concept}

In the CAPLOC project, we propose to enhance localization performances in particular accuracy, by knowing the structure of the environment crossed by the vehicle. This structure can be obtained by images provided by several cameras installed on the roof of the vehicle. The approach has already been validated with the PREDISSAT tool (Marais 2005). PREDISSAT is, however, not a real time process and can largely benefit from new image processing improvements. This concept has been patterned by INRETS (now IFSTTAR) in 2006 (France) and 2008 (international) (Marais 2008). Figure 1 illustrates two kinds of representations of masking obstacles around the antenna. The left view is a polar view. The white area is the sky, the blue one is the masked zone. The right view is another representation of the same masked area drawing the elevation angle of the mask versus the azimuth.
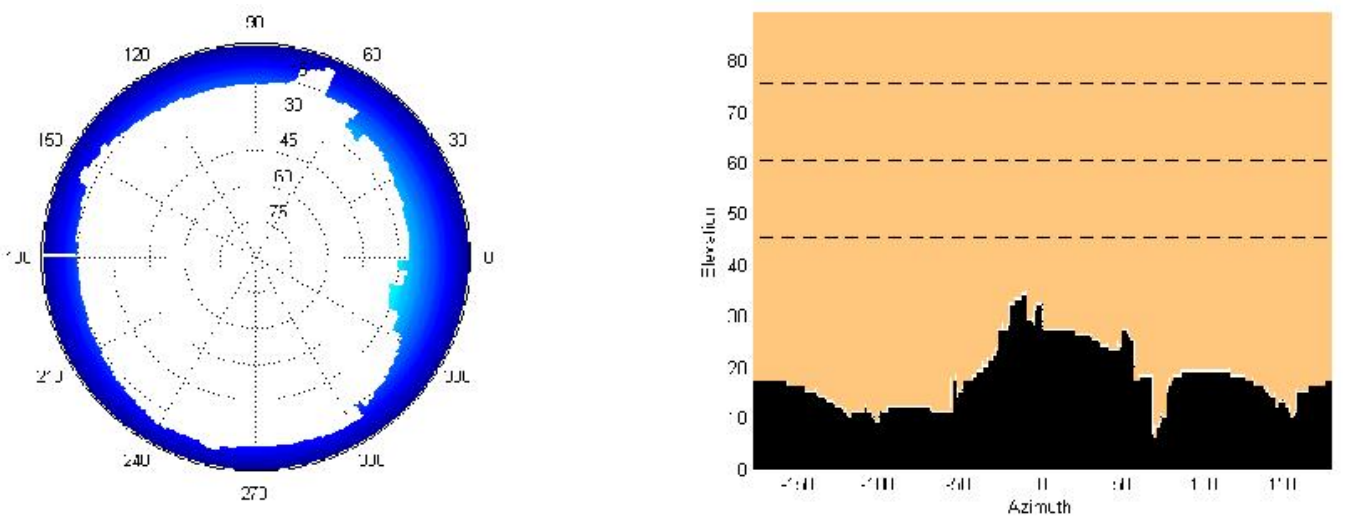

Fig. 1. Masking obstacles around the receiver antenna (left: sky view, right: panoramic view). These data have been obtained using the SE-NAV software (www.oktal-se.com/se-nav).

In a first step, the state of reception of satellite signals is determined by comparing satellite positions and obstacle positions around the antenna. Satellites positioned in the sky area are directly visible (LOS Line Of Sight), satellites positioned behind the mask are NLOS. In (Meguro 2008), the authors explore this way by calculating the visible sky area with an infrared camera. The most interesting obstacles are the bulkiest ones (buildings for example). The thinnest ones create local phenomena that will stay very shortly if the receiver moves and will not present major problems in our dynamic applications. Indeed, with this camera, the sky appears particularly dark, allowing easier to detect the boundary between the sky (dark) and the building (white). The major drawbacks of this approach are i) the high cost of this system and the difficulty to deploy it on a large scale; ii) a specific calibration, making once again the solution difficult to apply; iii) in case of overexposure, this type of camera can lead to errors of detection. (Obst 2012) and (Peyret 2011) use 3D models to identify satellite visibility. (Ramalingam 2010), (Cappelle, 2011) and (Koch 2007) combine the use of omnidirectional camera and 3D model of the environment and match some data between the acquired image and the 3D model. 
in : Transportation Research Part C: Emerging Technologies, 2013 @elsevier

http://www.sciencedirect.com/science/journal/0968090X

Another use of 3D models is presented by (Groves 2012) who compares satellite real visibility and theoretical visibility obtained with the model to refine lateral accuracy of the position or by (Bourdeau 2012) who includes a pseudo-range bias estimation in EKF.

The main disadvantage of 3D model-based approach concerns the difficulty to dispose of a precise 3D model of the environment. Furthermore, the real time constraint is not respected. This is also the case of our previous works on the characterization of the environment using an efficient segmentation strategy combining color and texture information (Meurie 2010), (Cohen 2009) and (Cohen 2010). Indeed, even if the results obtained with the proposed method are very satisfying, the processing time remains far too high. Like Meguro, we propose to identify sky in the acquired image by using a single camera delivering visible range to overcome the problems previously identified. The proposed real time strategy is based on a simplification of image (acquired in mobility) and a pixels classification (coupled with a post-treatment). The information extracted from the image will allow us to detect, then to exclude, the satellites which degrade the receiver performances, as the RAIM (Receiver Autonomous Integrity Monitoring) algorithm often does in aviation applications. However, these exclusion policies will often reduce drastically the availability in urban environments.

In a second step, out of the focus of this paper, we will develop a process of 3D model creation in order to model the environment structure around the antenna. This process has to be easy to use and compatible with the operational constraints of the transport operators. The goal is to obtain a 3D model with, if possible, only one run and the shortest mobilization of the vehicle. The process is composed of several tasks: the first one aims at defining a set of 3D points as dense and accurate as possible. Two main methods can be considered to compute the depth information: the first one uses images taken successively (Structure from Motion), and the second one uses images taken at the same time but from slightly different viewpoints (Stereovision). In CAPLOC project, the stereovision approach is chosen to compute 3D points (Moreau 2012). The second task consists in determining a mesh from the 3D points previously computed. This model, associated to a ray tracing tool (Godefroy 2006), (Suh 2007) will allow us to calculate, and then correct, the pseudo-range error induced by the signal reflections.

\section{Image Processing strategy}

In this section, an image processing strategy to detect visible sky in acquired images is presented. The aim of this strategy consists in determining satellites that are located in sky regions (satellites received with a direct signal) and those located in non-sky regions (satellites with a blocked/reflected signal). This satellite classification will allow to exclude or weight the influence of these satellites in the new position computation, as explained in the next section. Figure 2 illustrates several images (5 images / 170) acquired with the laboratory vehicle (equipped with a fisheye camera installed on the roof and oriented upwards to capture images of the sky, and two GNSS receivers). This experimental platform permits to acquire real data when traveling in different types of environments and then to evaluate our algorithms considering on-board constraints such as real time constraint, overexposure, brightness changes, etc.
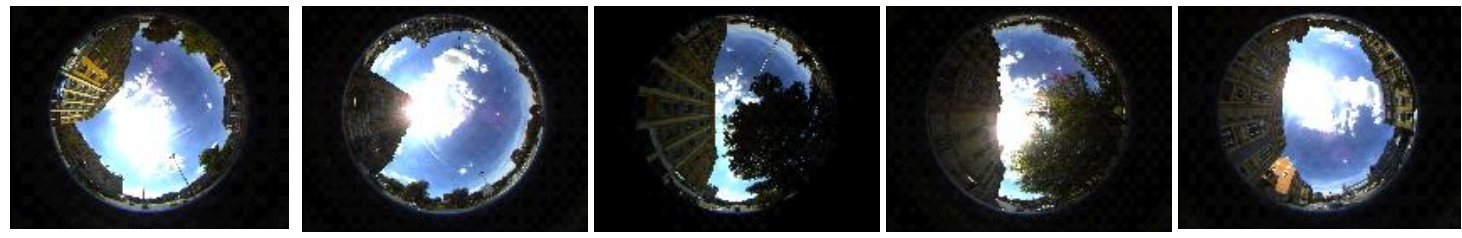

Fig 2: Five images of the database (acquired in the CAPLOC project) with varying complexity (overexposure, brightness changes). 
in : Transportation Research Part C: Emerging Technologies, 2013 @elsevier

http://www.sciencedirect.com/science/journal/0968090X

Considering the image processing phase, many segmentation and classification methods of literature could be considered. However, most of them do not allow to obtain satisfactory results in the context of this application. For example, (Chowdhury 1998) presents an automatic building and sky detection system based on texture features in color images taken in urban environments. This method seems to require high computational time and provide inaccurate results. In our previous works (Meurie 2010), (Cohen 2010), a color and texture based segmentation technique has been proposed. Combined with kmeans based classification, this technique provides a very good classification rate of $97.4 \%$, compared to a reference classification. Nevertheless, the processing time of this technique is incompatible with the real time constraint imposed by the application. Therefore, a new strategy fully respecting this constraint is proposed and presented below. Figure 3 illustrates the synopsis of the global strategy, which is composed of eight steps: data acquisition phase, five image processing steps, evaluation phase, and calculation of a new position.

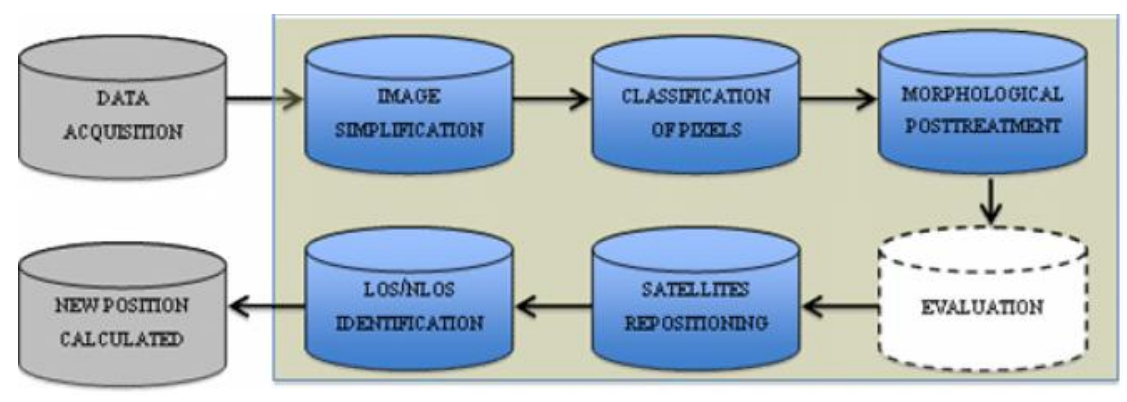

Fig 3: Synopsis of the image processing-based method to exclude/weight the influence of satellites for new position calculation.

Once data have been acquired, the first step of image processing consists in simplifying the acquired image using a geodesic reconstruction by dilatation (RGD) with an optimum parameter of contrast $\mathrm{H}$. The parameter value is different according to the classifier and the image database used, but it is optimized from a series of experimental tests. The choice of the geodesic operator is justified by its effect on the grayscale distribution of the sky and the good results compared to those obtained with other filters (Gaussian, Median, Mean, Nagao). Indeed, images have been acquired in different conditions (illumination changes, overexposure, shadows, etc.), and it is important to homogenize sky regions by simplifying the images.

The second step consists in classifying all pixels into two classes (sky and non-sky). In previous works (Attia 2011), the authors have compared the performance of different clustering algorithms on a smaller database. For that, several unsupervised (Fisher, KMlocal, Fuzzy-Cmeans, SRM) and supervised (Bayes, K-ppv, SVM) classifiers are tested, with an appropriate value of the parameter $\mathrm{H}_{\mathrm{RGD}}$. In this paper, the database is more important and the classification step is combined with a morphological post-treatment that consists in reclassifying incoherent regions for which the area is less than 27500 pixels (ie. a small region considered as sky but located in a non-sky/building region). Table 1 illustrates the classification results and the computational time according to the classifier and the parameters used. One can notice that Fisher algorithm gives the best classification rate (labelled $\mathrm{C}_{1}$ in Table 1) compared to other unsupervised classifiers (95.8\% with Fisher VS 94.7\% with KMlocal VS 94.6\% with $75.7 \%$ with SRM). Considering supervised classifiers, Kppv algorithm gives the best results (97.4\% VS 90.3\% with Bayes VS 94.7\% with SVM). Table 1 also illustrates the need for the simplification (labelled $\mathrm{C}_{2}$ in Table 1 ) and the post- 
in : Transportation Research Part C: Emerging Technologies, 2013 @elsevier

http://www.sciencedirect.com/science/journal/0968090X

6

treatment (labelled $\mathrm{C}_{3}$ in Table 1) steps. Indeed, the simplification step increases (from $+0.4 \%$ to $21.3 \%$ compared with $\mathrm{C}_{1}$ ) the classification results obtained without this step. The combination of the simplification, the classification and the post-treatment steps (labeled $\mathrm{C}_{3}$ in Table 1) also increases the global classification rate (until $+2.5 \%$ compared with $\mathrm{C}_{2}$ ). In conclusion, one can notice that a strategy based on simplification by RGD coupled with a classification with Fisher's algorithm and a posttreatment gives the best compromise between quality of results and computational time (a good classification rate equal to $97.2 \%$ with a processing time of $37 \mathrm{~ms}$ ).

\begin{tabular}{|c|c|c|c|c|c|c|}
\hline Algorithm & Parameters & $\begin{array}{l}\text { Classification } \\
\qquad\left(\mathrm{C}_{1}\right)\end{array}$ & $\begin{array}{c}\text { Simplification } \\
+ \\
\text { Classification } \\
\left(\mathrm{C}_{2}\right)\end{array}$ & $\mathrm{H}_{\mathrm{RGD}}$ & $\begin{array}{c}\text { Simplification } \\
+ \\
\text { Classification } \\
+ \\
\text { Post-treatment } \\
\left(\mathrm{C}_{3}\right) \\
\end{array}$ & Time (s) \\
\hline \multirow{4}{*}{ Fisher } & Red & $81.2 \%$ & $89.6 \%$ & 100 & $91,6 \%$ & \multirow{4}{*}{0.037} \\
\hline & Green & $89.9 \%$ & $93.5 \%$ & 50 & $93.9 \%$ & \\
\hline & Blue & $95.8 \%$ & $96.4 \%$ & 25 & $97.2 \%$ & \\
\hline & Average & $91.4 \%$ & $93.6 \%$ & 50 & $94.1 \%$ & \\
\hline \multirow{4}{*}{ KMlocal } & EZHybrid & $94.7 \%$ & $95.2 \%$ & 25 & $96.4 \%$ & \multirow{4}{*}{0.45} \\
\hline & Hybrid & $94.7 \%$ & $95.2 \%$ & 25 & $96.3 \%$ & \\
\hline & Loyds & $94.7 \%$ & $95.2 \%$ & 25 & $96.5 \%$ & \\
\hline & Swap & $94.3 \%$ & $95.4 \%$ & 25 & $96 \%$ & \\
\hline \multirow{3}{*}{$\begin{array}{l}\text { Fuzzy- } \\
\text { Cmeans }\end{array}$} & Fuzzyness=1 & $95.2 \%$ & $95.2 \%$ & 25 & $96.2 \%$ & \multirow{3}{*}{3.34} \\
\hline & Fuzzyness $=1.5$ & $94.4 \%$ & $95.4 \%$ & 25 & $97.5 \%$ & \\
\hline & Fuzzyness=2 & $94.6 \%$ & $95.4 \%$ & 25 & $97.9 \%$ & \\
\hline SRM & - & $75.7 \%$ & $97 \%$ & 100 & $97 \%$ & 0.47 \\
\hline Bayes & - & $90.3 \%$ & $96 \%$ & 100 & $97.3 \%$ & 0.47 \\
\hline \multirow{2}{*}{ Kppv } & $\mathrm{Nb} \_\mathrm{Kppv}=4$ & $97.4 \%$ & $97.8 \%$ & 25 & $98 \%$ & \multirow{2}{*}{15.4} \\
\hline & $\mathrm{Nb} \_K p p v=6$ & $97.4 \%$ & $98 \%$ & 25 & $98.1 \%$ & \\
\hline SVM & - & $94.7 \%$ & $97.1 \%$ & 25 & $97.1 \%$ & 5.04 \\
\hline
\end{tabular}

Table 1: Classification rate and computational time according to the classifier/parameter used.

The fourth step consists in satellite repositioning in the acquired image (cf. middle and right images of figure 4). On the one hand, the NMEA frames available at the output of every GPS receiver give the identity and position of every satellite receivable in an open sky environment. The position is then known in terms of azimuth and elevation. On the other hand, the fish-eye image, synchronized with the GPS receiver via the RTMaps software, can be seen as a polar target where satellite positions can be drawn. A 
calibration step is however required in order to take into account the distortion caused by the lens. This work has been performed on an experimental protocol.

The last step of image processing consists in identifying which satellite is located in a sky region (received with a direct signal) and in a non-sky region (with blocked/reflected signals). For a better visualization, figure 4 illustrates, for five images (among 170 images) of the database, the state of the satellite: a satellite with a direct signal, and respectively one with blocked/reflected signals, is colorized in green (respectively in red or blue). These data will allow us to weight the influence of some satellite in the calculation of the new position or exclude them from the solution. In this paper, the NLOS satellite exclusion policy will be applied.
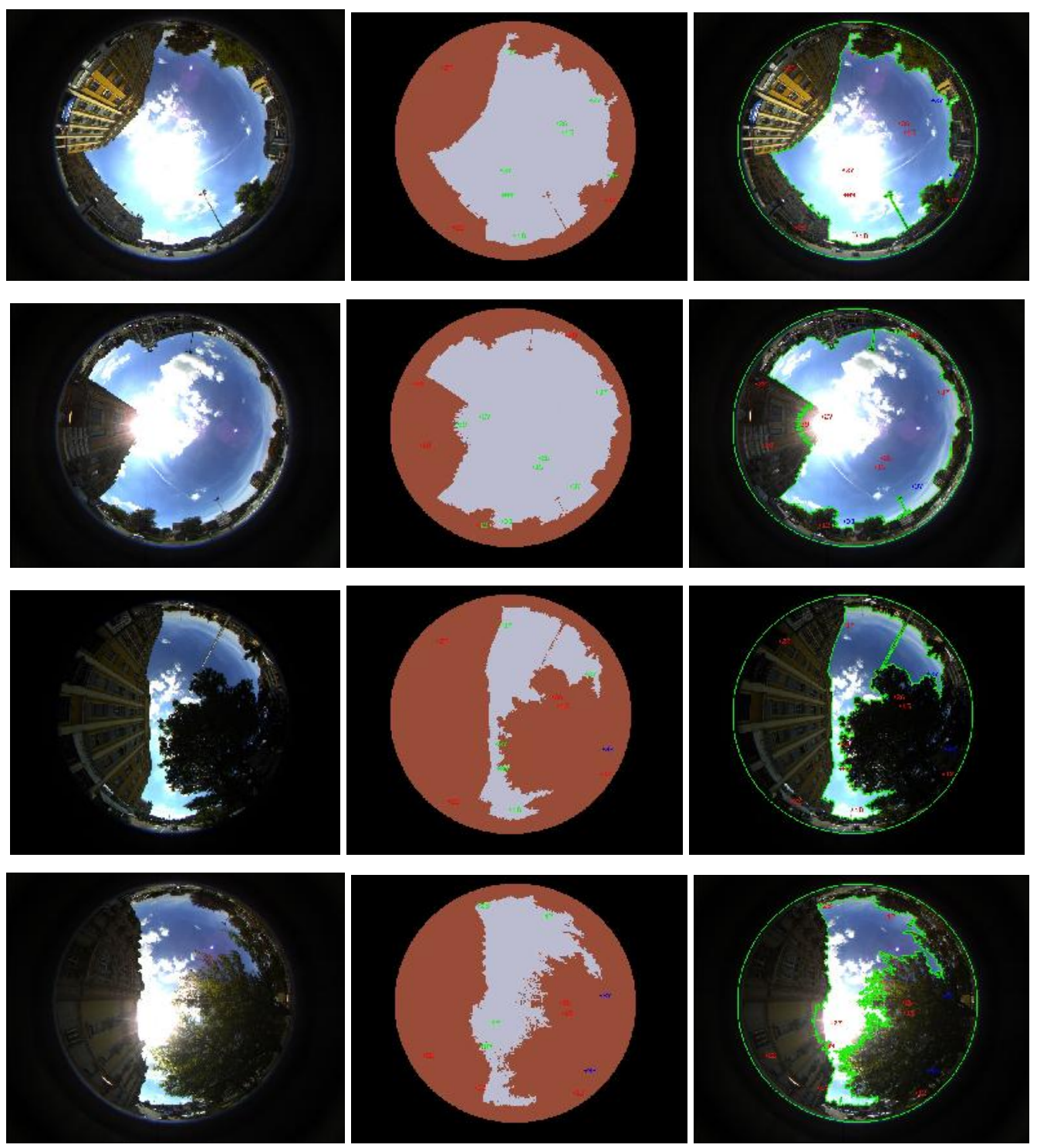

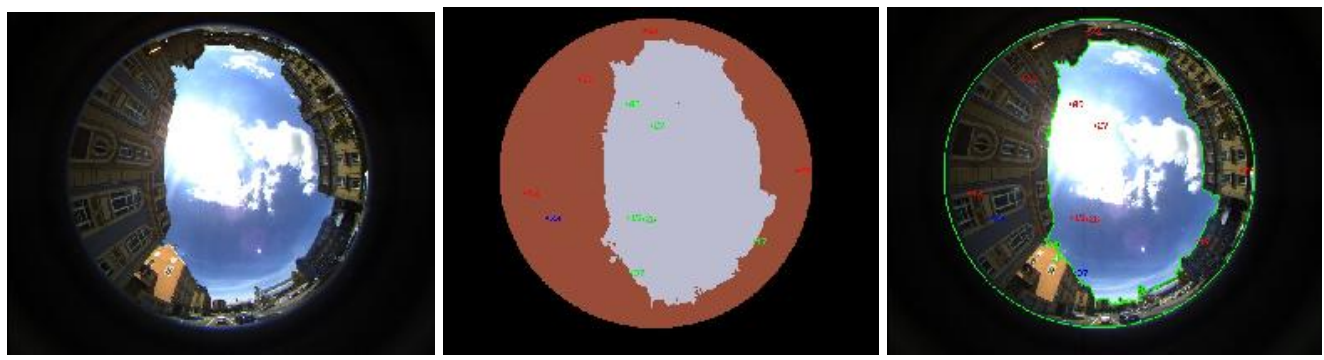

Fig. 4. Results of the image processing (left to right: acquired image, classified image into two classes (sky and non-sky) with satellites projection and the calculated horizon line).

\section{Satellite-based localization algorithms}

Satellite-based localization relies on the use of 4 satellite signals simultaneously received and on estimating their time of arrival (TOA). Accuracy will be the result of a good estimation of pseudorange measurements, but also good geometry of available satellites. Unfortunately, in transport environments TOA can be degraded by the propagation conditions in the receiver antenna surroundings, and induce inaccuracy in the positioning. To detect such events, our approach aims to identify reflected signals thanks to the environment description provided by a computer vision system. This technique can be compared to (Meguro 2008) based on infrared camera. In a same objective, (Peyret 2011) and (Obst 2012) propose to use $3 \mathrm{D}$ models in order to identify NLOS satellites. Once detected, these signals will be considered as failures and excluded from the solution computation.

Experimental results presented in this section take place in the CAPLOC project, started in October 2010. This section aims to demonstrate CAPLOC ability to exclude NLOS satellites.

The results presented hereafter use data recorded in the city of Belfort (real GPS data recorded with an uBlox receiver implemented in a Safedrive receiver), a GPS-RTK and a fisheye camera. Figure 5 illustrates the global trajectory of the vehicle in Belfort (in France). 


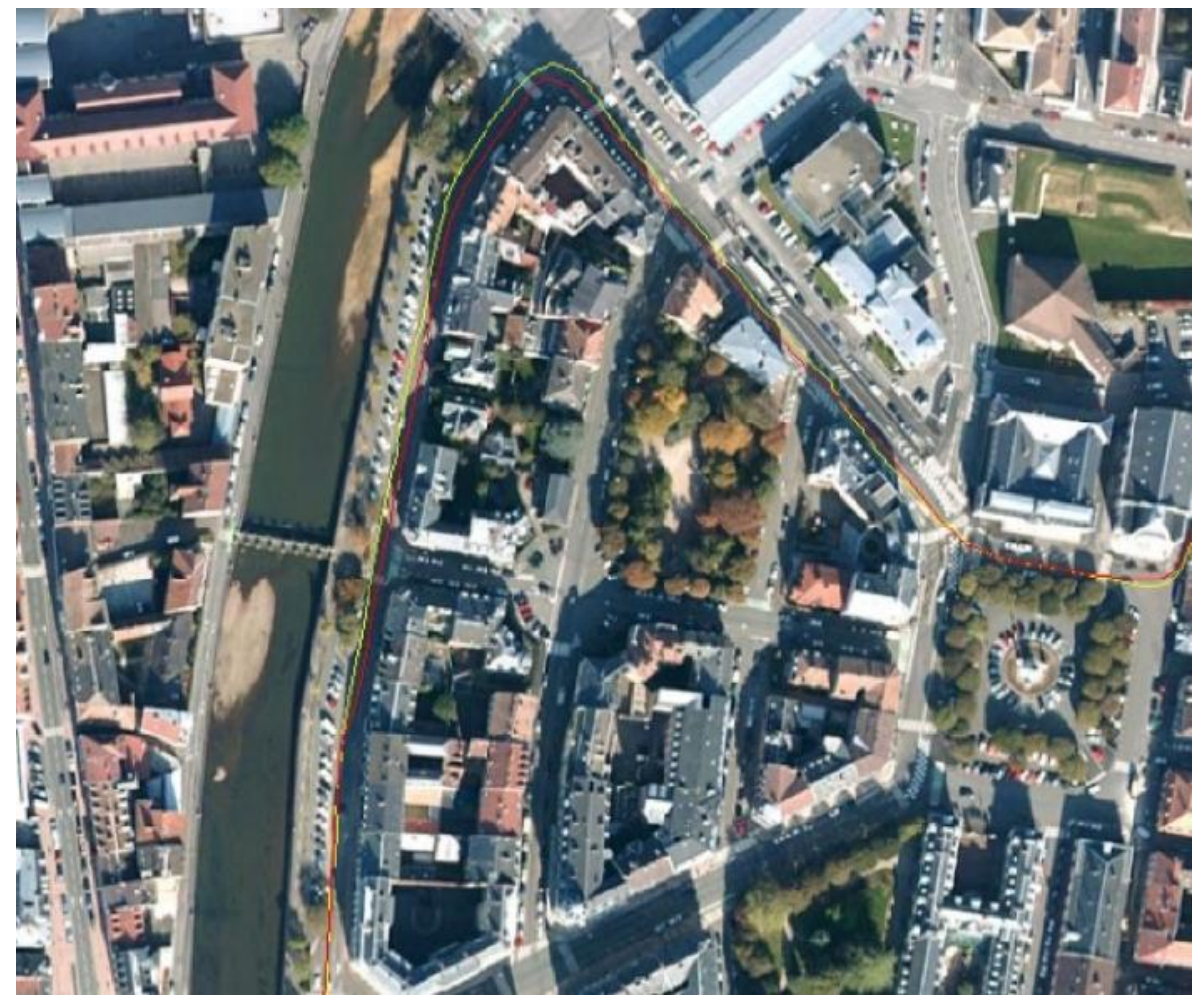

Fig 5: Example of one path of the vehicle in Belfort (the calculated position in yellow and the reference in red)

In the following subsections, we compare the effect of LOS/NLOS discrimination on positioning performances. Two approaches are tested: the first one consists in detecting NLOS satellites based on their reception power while the second one uses image processing as described above. For both, raw pseudorange measurements are used in a classical Extended Kalman Filter (EKF) in order to compare 2D positioning results with a reference (ground truth) resulting from measurements performed with a GPSRTK receiver. For each method, the proposed solution, keeping only LOS signals, is compared with the solution obtained with all the received satellites. In figures 6 and 7, the red line corresponds to reference. The blue and the green lines are respectively obtained with all the received satellites and with only LOS satellites.

\subsection{Results based on a CNO threshold}

CN0 characterizes Carrier-to-Noise ratio (CN0). It decreases when signal is attenuated by propagation effects like reflections on obstacles. The threshold between LOS and NLOS signal CNO is difficult to determine once for every campaign and equipments. Indeed, mean CN0 will vary function of the receiver, antenna and satellite elevation. For this experimentation, we have chosen a first threshold equal to $42 \mathrm{~dB}$ based on (Viandier 2011) and a second one of 40dB from the graphs presented in (Wang 2007). This value corresponds to the mean CN0 received for LOS satellites with an elevation of 10 degrees. However, in the following, higher thresholds ( 44 and $46 \mathrm{~dB}$ ) have also been tested in order to evaluate its impact.

Figure 6 illustrates a vehicle's trajectory in Belfort. The position is calculated with a classical EKF, using all the available satellites (blue points) or only the LOS ones (green points). LOS signals are detected 
in : Transportation Research Part C: Emerging Technologies, 2013 @elsevier

http://www.sciencedirect.com/science/journal/0968090X

10

when their CNO is bigger than $42 \mathrm{~dB}$. Results are compared to ground truth, measured with a RTK receiver (red points). Accuracy is computed by projecting each position orthogonally on the reference trajectory. With all the satellites used, the mean error is equal to $13 \mathrm{~m}$. With exclusion of the supposed NLOS satellites, the error is reduced to $4.57 \mathrm{~m}$. One can notice that some outliers still remain but the global result is positive.

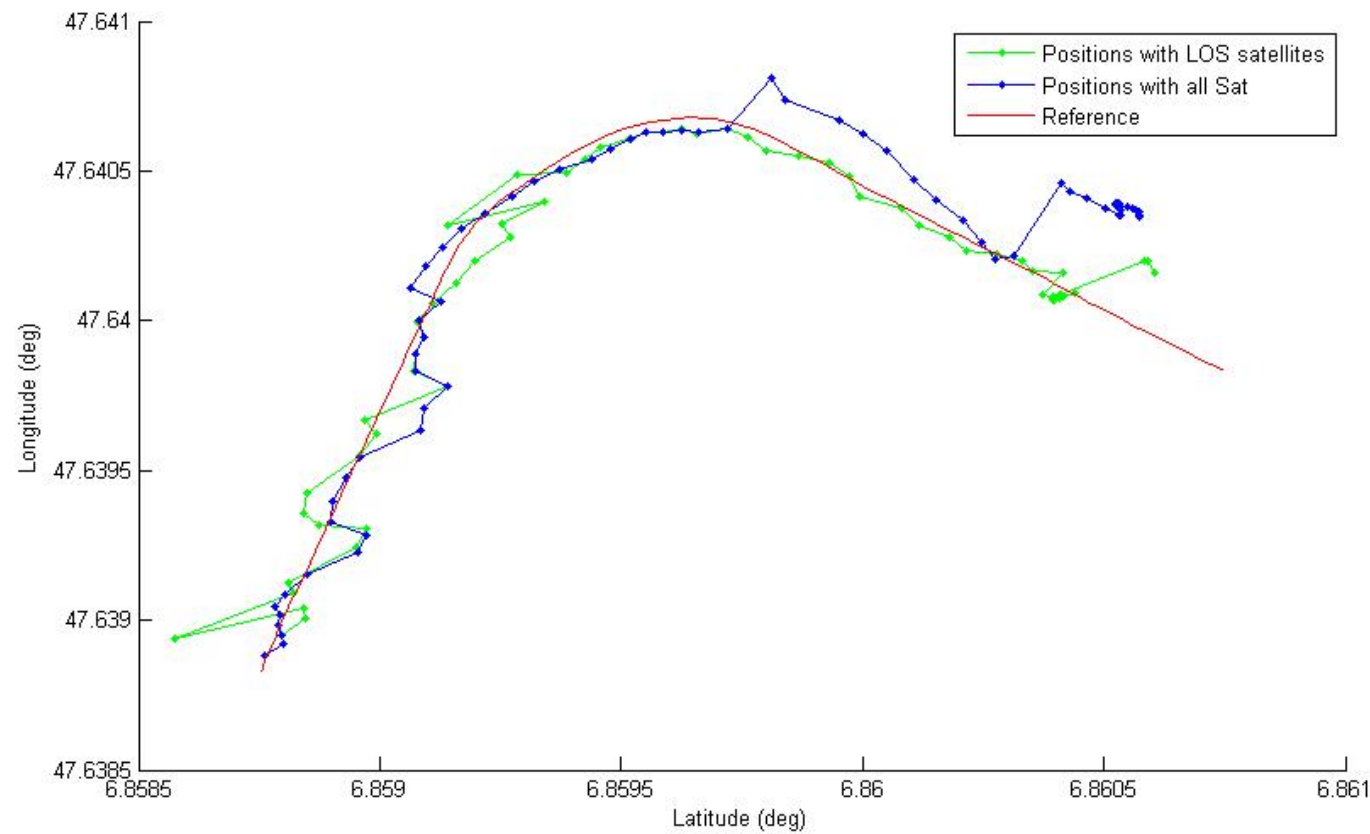

Fig. 6. View of the vehicle's trajectory in Belfort. The position is calculated with a classical EKF, using all the available satellites (blue points) or only the LOS ones (green points). LOS signals are detected when their CN0 is bigger than 42dB. The results are compared to the ground truth measured with a RTK receiver (red points).

As indicated above, other CNO thresholds have been tested in order to evaluate the impact of its choice on the performance. Results are summarized in table 2 . The highest the threshold is, the more satellites are excluded. One can notice that a high threshold (44dB or $46 \mathrm{~dB}$ ) enhances unavailability of the solution (3 and 9 points respectively for 71 measures) and increases the mean error. This is the result of the poor geometry of the resulting satellites configuration after exclusion. As expected, the value of $42 \mathrm{~dB}$ seemed to show the best result in our configuration but the result is close to the one obtained with $40 \mathrm{~dB}$. To avoid being dependent on the choice of this threshold, the image-based processing is tested.

\begin{tabular}{lccccc}
\hline & $\begin{array}{c}\text { With all } \\
\text { satellites }\end{array}$ & $\begin{array}{c}\text { LOS only } \\
(\mathrm{CN} 0>40 \mathrm{~dB})\end{array}$ & $\begin{array}{c}\text { LOS only } \\
(\mathrm{CN} 0>42 \mathrm{~dB})\end{array}$ & $\begin{array}{c}\text { LOS only } \\
(\mathrm{CN} 0>44 \mathrm{~dB})\end{array}$ & $\begin{array}{c}\text { LOS only } \\
(\mathrm{CN} 0>46 \mathrm{~dB})\end{array}$ \\
\hline Mean error $(\mathrm{m})$ & 13,12 & 4,58 & 4,57 & 6,32 & 24,79 \\
\hline Max error $(\mathrm{m})$ & 33,05 & 18,58 & 22,05 & 55,65 & 64,25 \\
\hline Standard deviation & 12,74 & 4,58 & 4,42 & 7,75 & 17,89 \\
\hline Unavailable points & 0 & 0 & 0 & 3 & 9 \\
\hline
\end{tabular}

Table 2. Performance comparison with the different approaches for NLOS satellite exclusion. 


\subsection{Results based on image-based characterization}

Figure 7 draws the same view than figure 6 but with an image processing-based solution. The red and blue points are the same than previously. Every satellite detected in a non-sky area by image processing is excluded from the EKF computation. Satellites received through vegetation are considered as NLOS thus excluded too. Here, with exclusion of the supposed NLOS satellites, error is equal to $11.47 \mathrm{~m}$. The improvement is not very convincing compared to the $13 \mathrm{~m}$ of the initial solution. The standard deviation is equal to $12.25 \mathrm{~m}$ and 6 points are unavailable. Nevertheless, several assumptions are studied in the next paragraph in order to propose evolutions of this strategy.

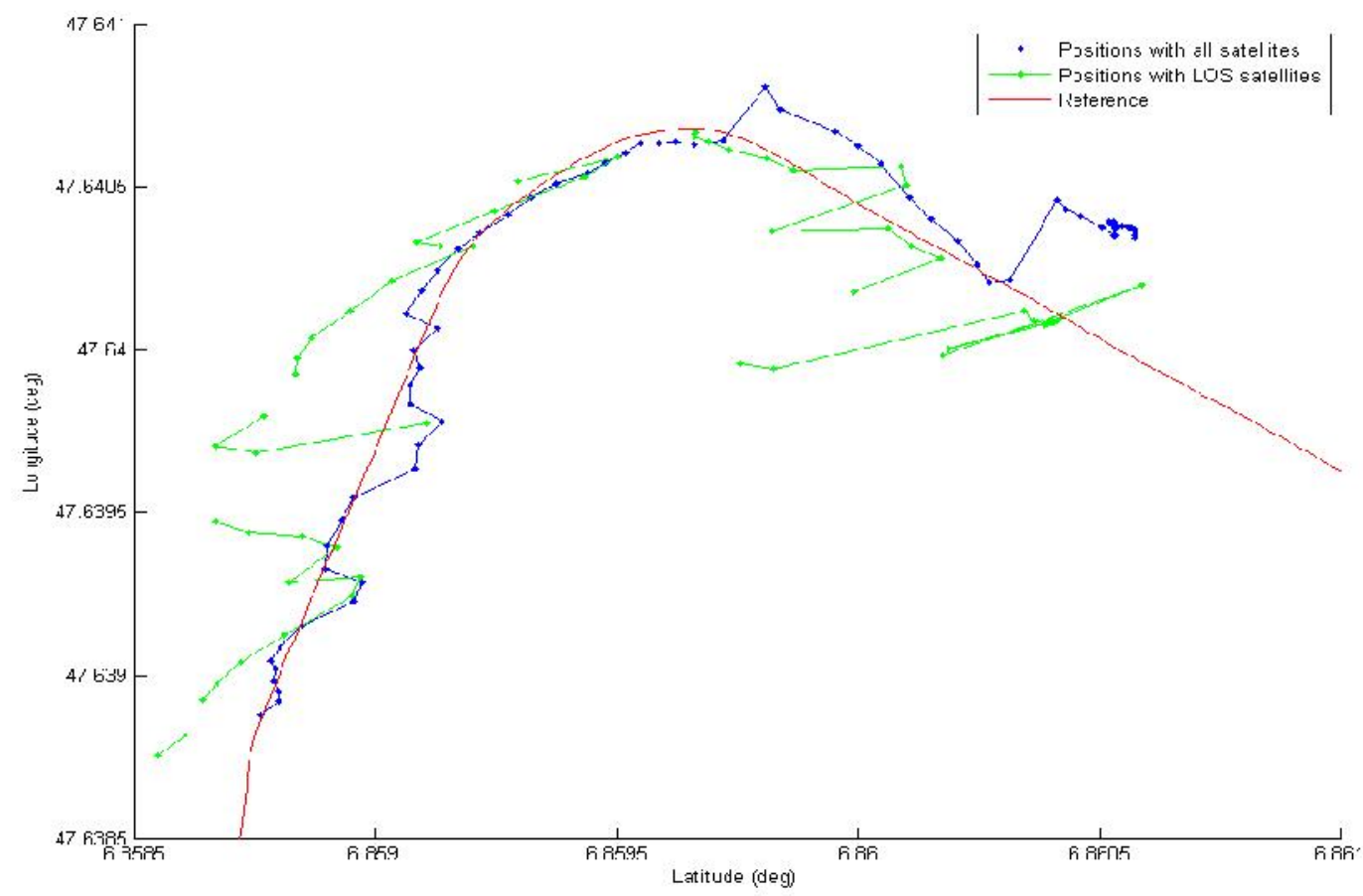

Fig. 7. View of the vehicle's trajectory in Belfort. The position is calculated with a classical EKF, using all the available satellites (blue points) or only the LOS ones (green points). LOS signals are detected when the satellite is placed in a sky area of the fish-eye image. The results are compared to the ground truth measured with a RTK receiver (red points).

\subsection{Critical analysis}

The two approaches described above apply different LOS/NLOS detection techniques but exclusion process is the same as well as EKF applied. Comparing the two detection methods, one can conclude that CNO-based threshold enhances much better accuracy than our image-based solution. Figure 8 presents the positioning error with the three solutions: all satellites (in blue), CN0-based and image-based detection (in green and red).

The first part of the trajectory is clearly improved by our image-based exclusion procedure ( 0 to $20 \mathrm{~s}$ ). This period corresponds to a vehicle stop, strongly disturbed by multipath. In such a configuration, image-based NLOS detection and exclusion is an added-value for accuracy. Last part of the run (45s to 
in : Transportation Research Part C: Emerging Technologies, 2013 @elsevier

http://www.sciencedirect.com/science/journal/0968090X

12

70s) is degraded when exclusion is applied, especially with image-based classification. In Figure 5 Google Earth view of the path shows clearly the configuration of the scene. Half of the street is almost an open-sky environment and other half is bordered by building. Thus, exclusion occurs on satellites positioning on the right hand side of the trajectory (considering vehicle running from the top to the bottom in the figure). As a consequence, DOP reaches the value of 7 , which characterizes a very poor geometry of the satellites and poor accuracy.

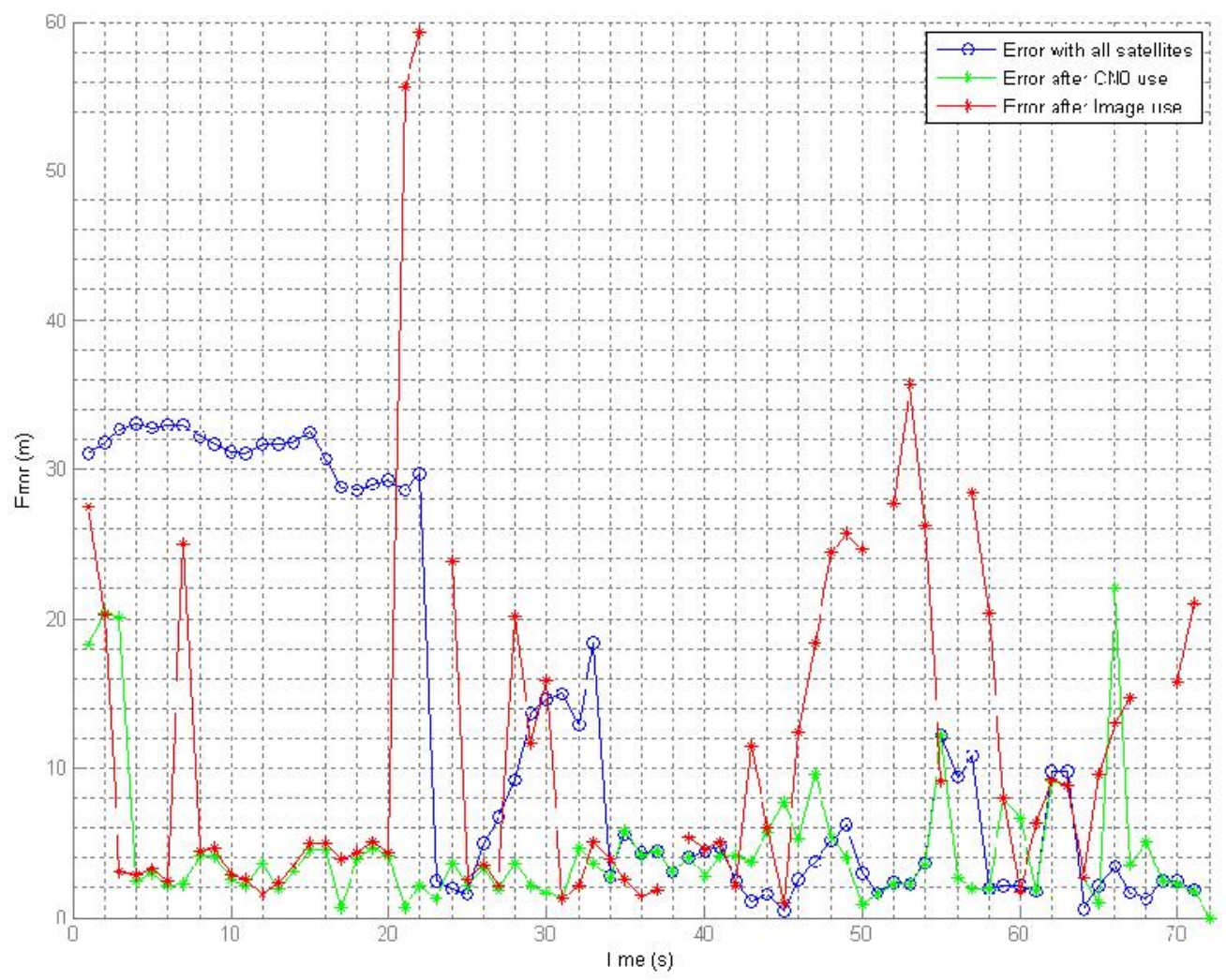

Fig. 8. Comparison of the errors obtained with a typical EKF applied on all the satellites (blue curve), LOS - received with a CN0 $>42 \mathrm{~dB}$ (green curve) and LOS - received in a sky area of the image (red curve). 


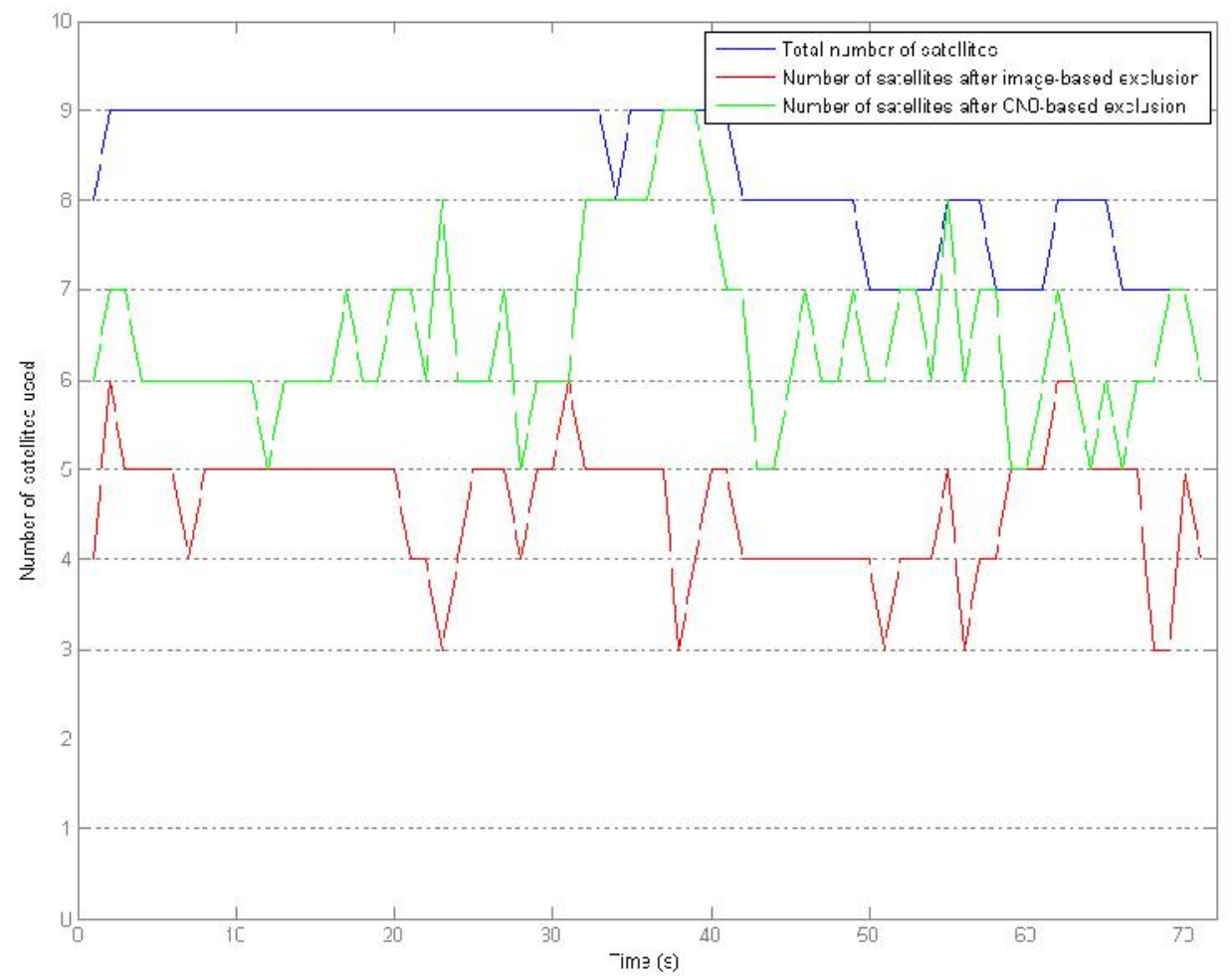

Fig. 9. Comparison of the number of satellites kept for the EKF. The total available satellites is represented by the blue curve; the number of satellites after $\mathrm{CN} 0$ exclusion in on the green curve and after image detection in red.

Figure 9 shows the total number of satellites received (blue line) and after exclusion (with CNO in green, and with image detection in red). The severity of the image-based detection is clearly visible compared to CNO. Analysis of these values allows us to propose perspectives to enhance efficiency of the image use. First, considering vegetation as a blocking obstacle might be too restrictive. As done in Land Mobile Satellite channel models (Akturan 1997), (Bråten 2002), we could consider a third intermediate state. Second, one should study if satellites positioned at an elevation close from the horizon line should be considered as LOS based on diffraction contribution or considering inaccuracy in the image calibration. Finally, a balance between DOP and LOS/NLOS detection will be studied.

\section{Conclusions}

In this paper, we have presented the CAPLOC project objectives and principles. The goal of such a project is to show the feasibility of an intelligent use of GNSS satellites in a harsh environment in order to provide a solution able to answer accuracy requirements of land transportation systems, road, railway or tramway. The concept relies on the detection of satellites received without any direct ray, called NLOS satellites. In this paper, two approaches for such detection have been presented. The first one is a classical detection considering CNO level. The result of such a sorting is convincing in this paper as we have tested our equipment before in order to choose an appropriate CN0 threshold. However, any change in the 
in : Transportation Research Part C: Emerging Technologies, 2013 @elsevier

http://www.sciencedirect.com/science/journal/0968090X

equipment will require a new calibration. The second method relies on the use of image processing and is based on detection of sky and non-sky areas. Results are mixed but promising. Indeed accuracy is increased, in particular when the vehicle is static. There multipath is well-detected and eliminated. In the rest of the trajectory, the image-based exclusion has been too severe and degrades accuracy because of a poor resulting geometry of the used satellites. The main advantage of such a solution is independency to any embedded 3D model that is required in some other papers and the lack of threshold choice (of CNO level for example). In the CAPLOC project, these objectives will be pursued. A next step will be the use of the knowledge offered by image to evaluate deterministically pseudorange errors created by obstacles in order to effectively use these NLOS received signals.

\section{Acknowledgements}

This work has been supported by the PREDIT (program of research, experimentation and innovation in land transport) and is funded by the French ministry MEDDE.

\section{References}

Akturan, R. , Vogel, W.J. (1997), Path diversity for LEO satellite-PCS in the urban environment, IEEE Trans. Antennas Prop., AP45, nº pp.1107-1116, July 1997.

Attia, A., Meurie, C., Ruichek, Y., Marais, J. (2011), Counting of satellites with direct GNSS signals using Fisheye camera. A comparison of clustering algorithms, IEEE International Conference on Intelligent Transportation Systems (ITSC), Washington, USA, October 2011.

Bourdeau, A., Sahmoudi, M., Tourneret, J.-Y. (2012), Tight integration of GNSS and a 3D city model for robust positioning in urban canyons, ION GNSS, Nashville Tennessee, September 2012.

Bråten, L.E., Amaya, C., Rogers, D.V. (2002), Statistical characterization of land mobile satellite propagation environments using a photogrammetric technique, URSI, 2002.

Cappelle, C., ·El Najjar, M.E., Charpillet F.,Pomorski, D. (2011), Virtual 3D City Model for Navigation in Urban Areas, J Intell Robot Syst, July 2011.

Chowdhury, I-N., (1998), Automatic detection of buildings and sky in color fisheye images, PhD Thesis, University of Texas at Austin, August 1998.

Cohen, A., Meurie, C., Ruichek, Y., Marais, J., Flanquart, A. (2009), Quantification of GNSS signals accuracy : an image segmentation method for estimating the percentage of sky. IEEE Internayinal confrenece on Vehicular Electronics and Safety (ICVES), pp. 40-45, 2009.

Cohen, A., Meurie, C., Ruichek, Y., Marais, J. (2010), Characterization of the reception environment of gnss signals using a texture and color based adaptive segmentation technique, IEEE Intelligent Vehicles Symposium (IV), pp. 275-280, San Diego, California, June 2010.

Godefroy, B., Sayerse, P. (2006), Amélioration du positionnement par satellites dans un environnement fortement urbanisé, grâce à la prédiction de la propagation des signaux GNSS, Revue Navigation, Vol. 54, n 213, pp. 17-35, jan. 2006.

Groves, P. D. (2011), Shadow matching: a new GNSS positioning technique for urban canyons, The journal of Navigation, 64, pp. 417-430, 2011. 
in : Transportation Research Part C: Emerging Technologies, 2013 @elsevier

http://www.sciencedirect.com/science/journal/0968090X

Koch, O., Teller, S. (2007), Wide-area egomotion estimation from known 3D structure. Con- ference on Computer Vision and Pattern Recognition (CVPR), 2007.

Kovář, P., Vejražka, F., Seidl, L., Puričer, P., (2005), Assessment of the EGNOS Signal Availability for Land Mobile User. 12th Saint Petersburg International Conference on Integrated Navigation Systems. Saint Petersburgh, pp. 148-156, May 2005.

Lentmaier, M., Krach, B., Robertson, P. (2008), Bayesian Time Delay Estimation of GNSS Signals in Dynamic Multipath Environments, Hindawi Publishing Corporation, International Journal of Navigation and Observation, Vol. 2008, Article ID 372651, 11 pages, 2008.

Marais, J., Ambellouis, S., Flancquart, A., Berbineau, M. (2008), Dispositif de localisation. French patent: WO/2008/040896, International patent: 10 avril 2008.

Marais, J., Berbineau, M., Heddebaut, M. (2005), Land Mobile GNSS, Availability and Multipath Evaluation Tool, IEEE Trans. On Vehicular Technology, Vol. 54, Issue 5, pp. 1697-1704, Sept. 2005

Meguro, J.-I., Murata, T., Takiguchi, J.-I., Amano, Y., Hashizume, T. (2009) GPS multipath mitigation for urban area using omnidirectional infrared camera, IEEE Trans. On Intelligent Transportation Systems, vol. 10, no. 1, pp. 22-30, Mar. 2009.

Meurie, C., Ruichek, Y., Cohen, A., Marais, J. (2010), An hybrid an adaptive segmentation method using color and textural information, IS\&T/SPIE Electronic Imaging 2010 - Image Processing: Machine Vision Applications III, Proc. of SPIE-IS\&T Electronic Imaging, SPIE Vol. 7538, 11 pages, California USA, January 2010.

Moreau, J., Ambellouis, S., Ruichek, Y. (2012), 3D reconstruction of urban environments based on fisheye stereovision, 8th International Conference on Signal Image Technology and Internet Based Systems, Italy, 2012.

Obst, M., Bauer, S., Wanielik, G. (2012), Urban multipath detection and mitigation with dynamic 3D maps for reliable land vehcile localization, PLANS, Monterey California, April 2012.

Pervan, B.S., Lawrence, D.G., Cohen, C.E., Parkinson, B.W. (1996), Parity space methods for autonomous fault detection and exclusion using GPS carrier phase, Position Location and Navigation Symposium, pp. 649-656, April 1996.

Peyret, F., Bétaille, D., Mougel, F. (2011), Non-Line-Of-Sight GNSS signal detection using an on-board 3D model of buildings, Proc. of the 11th international conference on ITS Telecommunications (ITST), St Petersburg, August 2011.

Ramalingam, S., Bouaziz, S., Sturm, P., Brand, M. (2010), Skyline2gps : Localization in urban canyons using omni-skylines, IEEE/RSJ International Conference on Intelligent Robots and Systems (IROS), 2010.

Suh, Y., Shibasaki, R., (2007), Evaluation of satellite-based navigation services in complex urban environments using a threedimensional GIS, IEICE Trans. Commun. Vol. E90-B, nº - July 2007.

Viandier, N. (2011), Modélisation et utilisation des erreurs de pseudodistances GNSS en environnement transport pour l'amélioration des performances de localisation, $\mathrm{PhD}$ Thesis from the Ecole Centrale of Lille, June 2011.

Wang, J.-H., Gao, Y. (2007), High-Sensitivity GPS Data Classification Based on Signal Degradation Conditions, IEEE Transactions on Vehicular Technology, Volume 56, Issue 2, pp. 566-574, March 2007. 